\title{
Факторы, влияющие на динамику качества жизни после хирургического лечения расслоения восходящего отдела и дуги аорты
}

\author{
Каменская О. В., Клинкова А. С., Логинова И. Ю., Чернявский А. М., Ломиворотов В. В., Караськов А. М.
}

Цель. Оценить влияние клинических и интраоперационных факторов на динамику качества жизни (КЖ) после протезирования аорты у пациентов с хроническим расслоением восходящего отдела и дуги аорты.

Материал и методы. В исследование включены 56 пациентов 50 (41-59) лет с хроническим расслоением аорты I типа по Дебейки. С помощью опросника SF-36 исследовалось KЖ до и в отдаленные сроки (через 12 мес.) после протезирования аорты. Методом многофакторного линейного регрессионного анализа проведена оценка факторов, оказывающих влияние на динамику различных параметров КЖ в отдаленном послеоперационном периоде. Результаты. До операции почти по всем параметрам опросника пациенты набрали от 52 баллов и ниже, что указывает на очень низкий исходный уровень КЖ. В отдаленные сроки после протезирования аорты выявлено статистически значимое улучшение следующих физических и психоэмоциональных показателей: ролевое функционирование, $(p=0,004)$; интенсивность боли, $(p=0,0001)$; жизненная активность, $(p=0,009)$; социальное функционирование, $(p=0,002)$; ролевое эмоциональное функционирование, $(p=0,009) ;$ физическое благополучие, $(p=0,02)$; душевное благополучие, $(p=0,03)$. При этом, не было отмечено положительной динамики по исходно низким параметрам оценки общего состояния здоровья и показателя психического здоровья.

По данным многофакторного регрессионного анализа, на динамику параметров КЖ в отдаленном периоде после протезирования восходящего отдела и дуги аорты влияют как интраоперационные условия (метод церебральной защиты), так и течение раннего послеоперационного периода (развитие неврологических осложнений, сердечно-легочной недостаточности, фибрилляции предсердий). Такие показатели, как пол, возраст, масса тела, сопутствующая патология, вид протезирования восходящего отдела аорты, время нахождения в отделении интенсивной терапии, время искусственной вентиляции легких не оказывают значимого влияния на КЖ в отдаленный период после операции

Заключение. Клиническими и интраоперационными факторами, неблагоприятно влияющими на динамику различных показателей КЖ в отдаленные сроки после протезирования восходящего отдела и дуги аорты, явились: церебральная защита в условиях глубокой гипотермии и краниоцеребральной гипотермии на фоне системной остановки кровообращения; развитие неврологических осложнений, а также фибрилляция предсердий и сердечно-легочная недостаточность в ранний послеоперационный период.
Российский кардиологический журнал. 2018;23(11):14-20

http://dx.doi.org/10.15829/1560-4071-2018-11-14-20

Ключевые слова: качество жизни, протезирование аорты, послеоперационные осложнения.

Конфликт интересов: не заявлен.

Финансирование. Работа выполнена при финансовой поддержке гранта РНФ № 17-75-30009.

ФГБУ Национальный медицинский исследовательский центр им. акад. Е. Н. Мешалкина Минздрава России, Новосибирск, Россия.

Каменская О.В. - д.м.н, В.н.с. группы клинической физиологии Центра анестезиологии и реаниматологии, ORCID: 0000-0001-8488-0858, Клинкова А. С. ${ }^{*}$ - к.м.н., н.С. группы клинической физиологии Центра анестезиологии и реаниматологии, ORCID: 0000-0003-2845-930Х, Логинова И. Ю. - к.б.н., с.н.с. группы клинической физиологии Центра анестезиологии и реаниматологии, ORCID: 0000-0002-3219-0107, Чернявский А. М. - д.м.н., профессор, руководитель Центра хирургии аорты, коронарных и периферических артерий, ORCID: 0000-0001-9818-8678, Ломиворотов В.В. - д.м.н., член-корр. РАН, руководитель Центра анестезиологии и реаниматологии, ORCID: 0000-00018591-6461, Караськов А.М. - д.м.н., академик РАН, заслуженный деятель науки РФ, директор, ORCID: 0000-0001-8900-8524.

*Автор, ответственный за переписку (Corresponding author): klinkovaas@ngs.ru

АПГМ - антеградная перфузия головного мозга, ИК - искусственное кровообращение, КЖ - качество жизни, ОК - остановка кровообращения.

Рукопись получена 27.12.2017

Рецензия получена 18.01.2018

Принята к публикации 25.01.2018

\section{Factors which influence the quality of life after surgical treatment of the dissection of ascending aorta and aortic arch}

Kamenskaya O. V., Klinkova A. S., Loginova I. Yu., Chernyavsky A. M., Lomivorotov V. V., Karaskov A. M.

Aim. To assess the impact of clinical and intraoperative factors on the dynamics of quality of life (QOL) after aortic prosthetics in patients with chronic dissection of ascending aorta and aortic arch.

Material and methods. The study included 56 patients (mean age 50 years) with chronic DeBakey type I aortic dissection. With the help of the SF-36 questionnaire, QOL was examined before and later (12 months) after aortic prosthetics. The method of multivariate linear regression analysis was used to evaluate factors that influence the dynamics of various parameters of $\mathrm{QOL}$ in the late postoperative period.

Results. Before the operation, patients scored from 52 points and below almost in all parameters of the questionnaire, which indicates a very low initial level of QOL. In the long-term period after aortic prosthetics, a statistically significant improvement in the following physical and psycho-emotional indicators was noted: role functioning $(p=0,004)$; bodily pain $(p=0,0001)$; vitality $(p=0,009)$; social role functioning $(p=0,002)$; emotional role functioning $(p=0,009)$; physical health $(p=0,02)$; mental health $(p=0,03)$. At the same time, there was no positive change in the initial low parameters of general health and psychiatric health perceptions.
According to multivariate regression analysis, the dynamics of QOL parameters in the long-term period after surgical treatment of the dissection of ascending aorta and aortic arch are affected by both intraoperative conditions (cerebral protection method) and the early postoperative period (neurological complications, cardiopulmonary failure, atrial fibrillation). Indicators such as gender, age, body weight, comorbidity, type of prosthesis of the ascending aorta, time spent in the intensive care unit, duration of artificial pulmonary ventilation do not have a significant effect on QOL in the long-term postoperative period.

Conclusion. Clinical and intraoperative factors that adversely affect the dynamics of various indicators of QOL in long-term periods after prosthetics of the ascending aorta and aortic arch were: cerebral protection in conditions of deep hypothermia and craniocerebral hypothermia against the background of systemic circulatory arrest; development of neurological complications, as well as atrial fibrillation and cardiopulmonary failure in the early postoperative period.

Russian Journal of Cardiology. 2018;23(11):14-20

http://dx.doi.org/10.15829/1560-4071-2018-11-14-20 
Key words: quality of life, aortic prosthetics, postoperative complications.

Conflicts of interest: nothing to declare.

Funding. This study was supported by the grant of the Russian National Fund (17-75-30009).

Meshalkin National Research Medical Center of RAS, Novosibirsk, Russia.

Хирургия аорты - один из самых интересных, сложных и драматичных вызовов современной сердечно-сосудистой хирургии. Наиболее тяжелый контингент - больные с расслоением аорты I типа по Де Бейки. Распространенность заболевания составляет 6 случаев на 100 тыс. населения в год, смертность не оперированных больных достигает $50 \%$ в первые 48 ч. Несмотря на тяжесть состояния, все пациенты с расслоением аорты I типа должны быть оперированы в срочном порядке. Операция позволяет снизить смертность в течение первого месяца, по сравнению с консервативным лечением, с 90 до $30 \%$ [1].

В нашем Центре внедрен в практику одноэтапный гибридный подход при хирургическом лечении расслоения аорты I типа по Де Бейки, что позволяет во время одного вмешательства восстановить анатомию аорты на ее значительном протяжении.

В последнее время уделяется особое внимание не только выявлению маркеров неблагоприятного исхода хирургического лечения расслоения аорты, а также определению факторов, влияющих на качество жизни (КЖ) данных пациентов в отдаленном послеоперационном периоде, так как уровень КЖ после операции характеризует ее эффективность [2]. К таким факторам можно отнести возраст, сопутствуюшую патологию, вид протезирования (с сохранением аортального клапана либо с применением клапансодержащего кондуита), интраоперационные условия, среди которых особое место занимают методы церебральной защиты, так как исход операции во многом зависит от сохранности центральной нервной системы [3-5]. Также на КЖ после операции могут повлиять различные осложнения, развившиеся в госпитальном периоде. Исследование КЖ подразумевает объективную и адекватную оценку состояния больного, его реакцию на лечение, эмоционально-психологический статус [6]. В связи с этим, изучение факторов, влияющих на динамику КЖ после хирургического лечения у пациентов с расслоением аорты, представляется актуальным.

Цель исследования - оценить влияние клинических и интраоперационных факторов на динамику КЖ после протезирования аорты у пациентов с хроническим расслоением восходящего отдела и дуги аорты.
Kamenskaya O.V. ORCID: 0000-0001-8488-0858, Klinkova A.S. ORCID: 00000003-2845-930X, Loginova I. Yu. ORCID: 0000-0002-3219-0107, Chernyavsky A. M. ORCID: 0000-0001-9818-8678, Lomivorotov V.V. ORCID: 0000-0001-8591-6461, Karaskov A. M. ORCID: 0000-0001-8900-8524.

Received: 27.12.2017 Revision Received: 18.01.2018 Accepted: 25.01.2018

\section{Материал и методы}

В проспективное когортное исследование включено 56 пациентов 50 (41-59) лет с диагнозом хроническое расслоение аорты I типа по Дебейки за период 2013-2015гг. Среди них 42 (75\%) больных мужского пола, 14 (25\%) - женского.

Причиной заболевания у 52 (93\%) пациентов был системный атеросклероз, у 4 (7\%) выявлен синдром Марфана.

Критерии включения:

1. наличие хронического расслоения аорты I типа по Дебейки с показаниями к оперативному лечению в виде протезирования восходящего отдела и дуги аорты;

2. наличие по данным компьютерной ангиографии замкнутой системы кровообращения головного мозга.

Критерии исключения:

1. гемодинамически значимое поражение брахиоцефальных артерий;

2. наличие неврологических нарушений;

3. наличие ранее перенесенных операций на сердечно-сосудистой системе, а также крупных полостных операций.

Исследование было одобрено локальным этическим комитетом, выполнено в соответствии со стандартами надлежащей клинической практики (Good Clinical Practice - GCP) и соответствовало принципам Хельсинкской декларации Всемирной медицинской ассоциации (ВМА). У всех пациентов было получено письменное информированное согласие.

Пациентам выполнялось протезирование восходящего отдела и дуги аорты как с сохранением аортального клапана (при отсутствии морфологических изменений полулунных створок и выраженного более 45 мм расширения корня аорты), так и с применением клапансодержащего кондуита. На первом этапе производилось протезирование восходящего отдела аорты в условиях искусственного кровообращения (ИК). По достижении необходимого уровня гипотермии приступали ко второму этапу операции - протезированию дуги аорты. На данном этапе в одном случае во время остановки кровообращения (OK) в качестве церебральной защиты применялась унилатеральная антеградная перфузия головного мозга (АПГМ) на фоне умеренной гипотермии (23$\left.24^{\circ} \mathrm{C}\right)$. При отсутствии технической возможности 
проведения АПГМ церебральная защита осуществлялась с помощью общей глубокой гипотермии $\left(18^{\circ} \mathrm{C}\right)$ и краниоцеребральной гипотермии (охлаждение головы матерчатым шлемом со льдом) на фоне системной ОК. Охлаждение пациента проводилось с температурным градиентом “теплоноситель-тело" 7-8 С. После завершения протезирования аорты пациент медленно согревался на фоне ИК до температуры в носоглотке $36^{\circ} \mathrm{C}$.

У всех больных до оперативного лечения и в отдаленные сроки после операции (через 12 мес.) оценивалось КЖ. Использовалась русская версия опросника SF-36, разработанного John E. Ware в Институте здоровья США [7]. Перевод на русский язык и апробация методики проведена "Институтом клинико-фармакологических исследований” (г. Санкт-Петербург). Пункты опросника сгруппированы в восемь шкал:

1. Physical functioning (PF) - физическое функционирование: отражает степень, в которой состояние здоровья лимитирует выполнение физических нагрузок (самообслуживание, ходьба, подъем по лестнице, переноска тяжестей и т.д.).

2. Role functioning physical (RF) - ролевое функционирование: влияние физического состояния на ролевое функционирование (работа, выполнение обычной деятельности).

3. Bodily pain (BP) - интенсивность боли и еe влияние на способность заниматься повседневной деятельностью.

4. General health perceptions $(\mathrm{GH})$ - общее состояние здоровья: оценка больным своего состояния в настоящий момент.

5. Vitality (VT) - жизненная активность: ощущение себя полным сил и энергии или, напротив, обессиленным.

6. Social functioning (SF) - социальное функционирование: определяется степенью, в которой физическое или эмоциональное состояние ограничивает социальную активность (общение).

7. Role functioning emotional (RE) - ролевое эмоциональное функционирование: предполагает оценку степени, в которой эмоциональное состояние мешает выполнению работы или другой повседневной деятельности.

8. Mental health (MH) - психическое здоровье: характеризует настроение (наличие депрессии, тревоги, общий показатель положительных эмоций).

Первые четыре шкалы отражают физический компонент здоровья, последующие четыре - психологическое здоровье пациента. Показатели шкал варьируют между 0 и 100, где 100 представляет полное здоровье. Результаты представляются в виде оценок в баллах. Все шкалы формируют два суммарных показателя: физическое и душевное благополучие Physical component score (PCS) и Mental component score (MCS).
Статистический анализ полученных результатов проведен с использованием пакета статистических программ Statistica 6.1 (USA). Данные представлены в виде $\mathrm{Me}\left(\mathrm{Q}_{25}-\mathrm{Q}_{75}\right)$, а также в численных значениях и процентах. Достоверность различий зависимых величин определяли по критерию Вилкоксона. С помощью многофакторного линейного регрессионного анализа проведена оценка факторов, оказывающих влияние на динамику различных параметров КЖ в отдаленном послеоперационном периоде с вычислением стандартизованного коэффициента регрессии ( $\beta)$. В многофакторный регрессионный анализ включали переменные, для которых значения критерия статистической значимости при однофакторном анализе составляли меньше 0,1. Достоверными принимали значения при уровне $\mathrm{p}<0,05$. Динамика $(\Delta)$ показателей КЖ вычислялась по формуле: ((параметр КЖ в отдаленные сроки после операции исходный параметр КЖ)/исходный параметр КЖ) *100\%.

\section{Результаты}

В таблице 1 дана клиническая характеристика и периоперационные данные пациентов с проксимальным расслоением аорты.

Среди сопутствующей патологии на первом месте у данных больных являлась гипертоническая болезнь, на втором и третьем местах ишемическая болезнь сердца и порок аортального клапана. В большинстве случаев пациентам выполнялось протезирование восходящего отдела аорты с сохранением аортального клапана.

В таблице 2 представлены послеоперационные данные и осложнения у пациентов с расслоением аорты.

В ранний послеоперационный период отмечено пять летальных исходов: у пациентов 49 и 54 лет в результате развития полиорганной недостаточности; у пациентки 44 лет в результате острого нарушения мозгового кровообращения и у двух пациентов 58 и 65 лет вследствие неконтролируемого кровотечения на фоне синдрома диссеминированного внутрисосудистого свертывания.

На рисунке 1 представлены значения шкал КЖ до и в отдаленные сроки после операции у пациентов с проксимальным расслоением аорты.

До операции почти по всем параметрам опросника пациенты набрали от 52 баллов и ниже, что указывает на очень низкий исходный уровень КЖ. Наиболее низкие показатели были выявлены по следующим шкалам: ролевое функционирование (RF), интенсивность боли (ВР), общее состояние здоровья (GH), жизненная активность (VT), ролевое эмоциональное функционирование (RE). По таким показателям, как PF и SF, затрагивающие сферы физического функционирования (самообслуживание, 
Таблица 1

\section{Клиническая характеристика и периоперационные данные пациентов с хроническим расслоением восходящего отдела и дуги аорты}

\begin{tabular}{|c|c|c|}
\hline \multicolumn{2}{|l|}{ Показатели, единицы измерения } & $\mathrm{n}=56$ \\
\hline \multirow{4}{*}{$\begin{array}{l}\text { Функциональный класс хронической сердечной } \\
\text { недостаточности по Нью-Йоркской классификации, n (\%) }\end{array}$} & 1 & $20(35,7)$ \\
\hline & II & $29(51,8)$ \\
\hline & III & $7(12,5)$ \\
\hline & IV & 0 \\
\hline \multicolumn{2}{|l|}{ Индекс массы тела (кг/м²), (Ме, 25-75\%) } & $27,9[21,3-31,1]$ \\
\hline \multicolumn{2}{|l|}{ Ишемическая болезнь сердца, n (\%) } & $10(17,9)$ \\
\hline \multicolumn{2}{|l|}{ Гипертоническая болезнь, n (\%) } & $44(78,6)$ \\
\hline \multicolumn{2}{|l|}{ Сахарный диабет, n (\%) } & $4(7,1)$ \\
\hline \multicolumn{2}{|l|}{ Порок аортального клапана, n (\%) } & $9(16,1)$ \\
\hline \multicolumn{2}{|l|}{ Фибрилляция предсердий, n (\%) } & $8(14,3)$ \\
\hline \multicolumn{2}{|l|}{ Хроническая обструктивная болезнь легких, n (\%) } & $3(5,4)$ \\
\hline \multicolumn{2}{|l|}{ Хроническая почечная недостаточность, n (\%) } & $6(10,7)$ \\
\hline \multirow[t]{3}{*}{ Супракоронарное протезирование восходящей аорты + } & $\begin{array}{l}\text { Протезирование дуги аорты путем наложения агрессивного косого } \\
\text { анастомоза, } \mathrm{n}(\%)\end{array}$ & $16(28,6)$ \\
\hline & Протезирование восходящего отдела и дуги аорты по типу “хобот слона”, n (\%) & $14(25)$ \\
\hline & Стентирование дуги аорты системой Djumbodis, n (\%) & $17(30,4)$ \\
\hline \multicolumn{2}{|c|}{$\begin{array}{l}\text { Протезирование аортального клапана и восходящего отдела аорты клапансодержащим кондуитом + протезирование дуги аорты путем } \\
\text { наложения агрессивного косого анастомоза, } \mathrm{n}(\%)\end{array}$} & $9(16,1)$ \\
\hline \multicolumn{2}{|c|}{$\begin{array}{l}\text { Церебральная защита при протезировании дуги аорты в виде антеградной унилатеральной перфузии головного мозга и умеренной } \\
\text { гипотермии, } \mathrm{n}(\%)\end{array}$} & $30(53,6)$ \\
\hline \multicolumn{2}{|c|}{$\begin{array}{l}\text { Церебральная защита при протезировании дуги аорты в виде краниоцеребральной гипотермии на фоне общей глубокой гипотермии, } \\
\text { n (\%) }\end{array}$} & $26(46,4)$ \\
\hline \multicolumn{2}{|l|}{ Время искусственного кровообращения (мин), (Ме, 25-75\%) } & $231,8[196,2-276,4]$ \\
\hline \multicolumn{2}{|l|}{ Время окклюзии аорты (мин), (Ме, 25-75\%) } & $138,7[111,3-185,4]$ \\
\hline
\end{tabular}

ходьба, подъем по лестнице) и социального функционирования (общение) пациенты набрали выше 70 баллов. Вышеизложенные данные свидетельствуют о влиянии патологии аорты как на физическую, так и на эмоциональную стороны жизни.

В отдаленные сроки после операции не вошли в исследование по КЖ семь человек: пятеро умерли в госпитальном периоде и двое через 8 и 9 мес. после операции в результате ишемического инсульта и острого инфаркта миокарда. За послеоперационный период наблюдения не было зарегистрировано осложнений со стороны сердечно-сосудистой системы (острый инфаркт миокарда, инсульт, повторные операции). Все пациенты выполняли рекомендации по послеоперационному обследованию и лечению.

В отдаленные сроки после протезирования аорты анализ уровня КЖ выявил статистически значимое улучшение следуюших физических и психоэмоциональных показателей: RF ( $p=0,004)$; BP $(p=0,0001)$; VT $(p=0,009) ; \operatorname{SF}(p=0,002) ; \operatorname{RE}(p=0,009) ;$ PCS $(\mathrm{p}=0,02) ; \mathrm{MCS}(\mathrm{p}=0,03)$. В данном случае не наблюдалось статистически значимой положительной динамики параметра физического функционирования (PF), отражающего способность выполнения наиболее простых повседневных физических нагрузок. Это
Таблица 2

\section{Послеоперационные показатели и осложнения у пациентов с хроническим расслоением восходящего отдела и дуги аорты}

\begin{tabular}{|l|l|}
\hline Показатели, единицы измерений & $\mathrm{n}=56$ \\
\hline Энцефалопатия, n (\%) & $5(8,9)$ \\
\hline Острое нарушение мозгового кровообращения, n (\%) & $4(7,1)$ \\
\hline Все неврологические осложнения, n (\%) & $9(16,1)$ \\
\hline Фибрилляция предсердий, n (\%) & $11(19,6)$ \\
\hline Легочно-сердечная недостаточность, n (\%) & $8(14,3)$ \\
\hline Почечная недостаточность, n (\%) & $7(12,5)$ \\
\hline Синдром полиорганной недостаточности, n (\%) & $5(8,9)$ \\
\hline Искусственная вентиляция легких (часы), Ме [25;75\%] & $48,4[25,3 ; 58,4]$ \\
\hline Искусственная вентиляция легких >24 ч, n (\%) & $14(25)$ \\
\hline Пребывание в отделении интенсивной терапии, (дни), & $4,2[3 ; 7]$ \\
\hline Ме [25;75\%] & $2(3,6)$ \\
\hline Острый инфаркт миокарда, n (\%) & $8(14,3)$ \\
\hline Инотропная поддержка >24 ч, n (\%) & $4(7,1)$ \\
\hline Рестернотомия, n (\%) & $5(8,9)$ \\
\hline Госпитальная летальность, n (\%) & $27,9[20 ; 37]$ \\
\hline Пребывание в стационаре, (дни), Ме [25;75\%] &
\end{tabular}

можно объяснить исходно высоким уровнем данного показателя (от 70 баллов и выше). При этом, не было отмечено статистически значимой положительной динамики по исходно низким параметрам оценки 


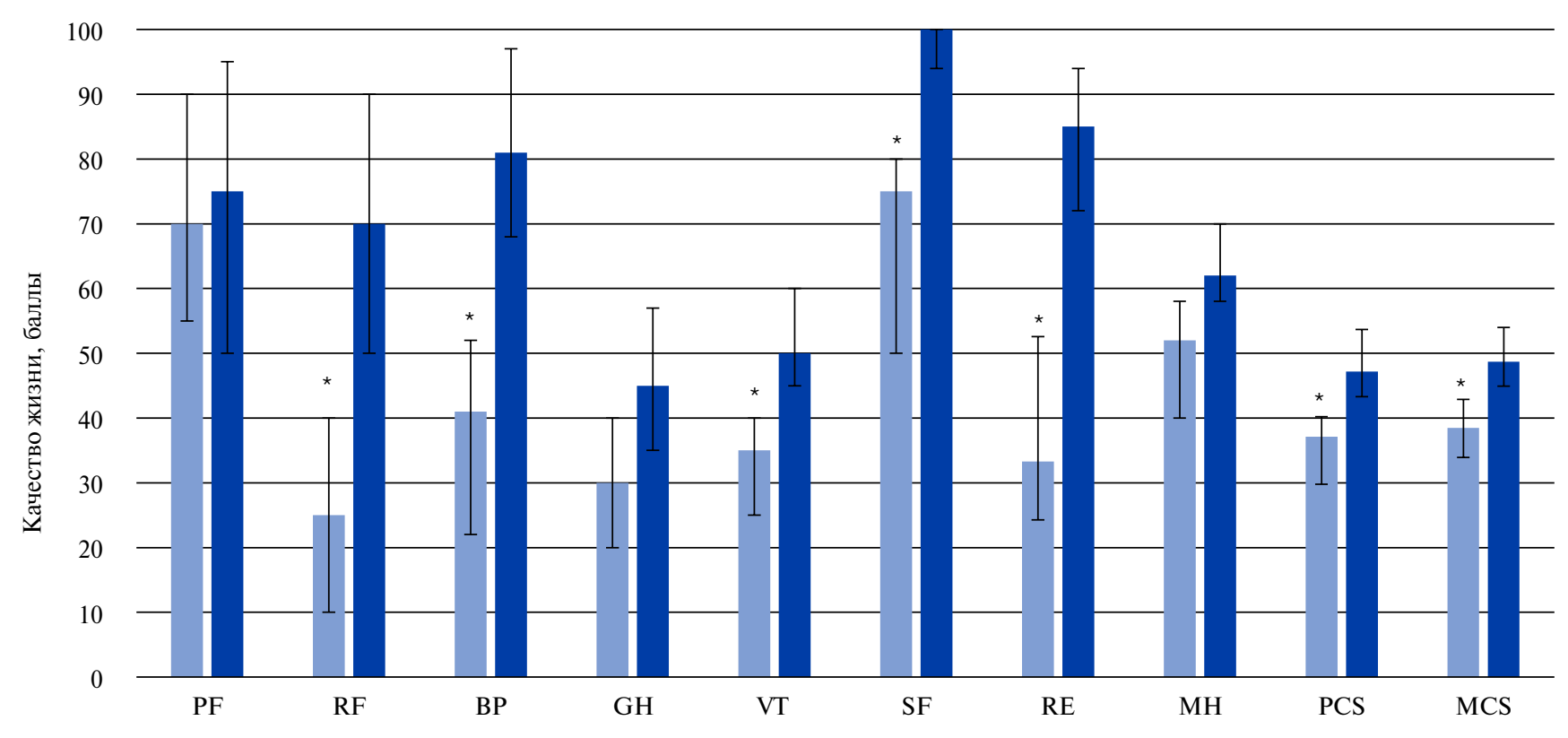

До операции

Отдаленные сроки после операции

Рис. 1. Распределение баллов согласно опроснику SF-36 до и в отдаленные сроки после протезирования восходящего отдела и дуги аорты. Примечание: * - статистически значимые различия с послеоперационными показателями $(p<0,05)$.

Сокращения: PF - показатели шкалы физического функционирования, RF - показатели шкалы ролевого функционирования, BP - показатели шкалы интенсивности боли, GH - показатели шкалы общего состояния здоровья, VT - показатели шкалы жизненной активности, SF - показатели шкалы социального функционирования, RE - показатели шкалы ролевого эмоционального функционирования, MH - показатели шкалы психического здоровья, PCS - показатели шкалы физического благополучия, MCS - показатели шкалы душевного благополучия.

Таблица 3

Факторы, влияющие на динамику показателей качества жизни в отдаленный период после протезирования восходящего отдела и дуги аорты (n=49)

\begin{tabular}{|c|c|c|c|}
\hline $\begin{array}{l}\text { Динамика }(\Delta) \text { показателей } \\
\text { КЖ }\end{array}$ & Предикторы (интраоперационный и ранний послеоперационный периоды) & $\begin{array}{l}\text { Коэффициент } \\
\text { регрессии ( } \beta)\end{array}$ & $\mathrm{p}$ \\
\hline PF & Сердечно-легочная недостаточность & $-1,044$ & 0,002 \\
\hline $\mathrm{RF}$ & $\begin{array}{l}\text { Сердечно-легочная недостаточность; } \\
\text { Фибрилляция предсердий }\end{array}$ & $\begin{array}{l}-0,595 \\
-0,395\end{array}$ & $\begin{array}{l}0,019 \\
0,060\end{array}$ \\
\hline $\mathrm{BP}$ & $\begin{array}{l}\text { Сердечно-легочная недостаточность; } \\
\text { Фибрилляция предсердий }\end{array}$ & $\begin{array}{l}-0,622 \\
-0,494\end{array}$ & $\begin{array}{l}0,001 \\
0,003\end{array}$ \\
\hline VT & $\begin{array}{l}\text { Сердечно-легочная недостаточность; } \\
\text { Фибрилляция предсердий }\end{array}$ & $\begin{array}{l}-0,571 \\
-0,767\end{array}$ & $\begin{array}{l}0,021 \\
0,023\end{array}$ \\
\hline SF & Сердечно-легочная недостаточность & $-0,860$ & 0,002 \\
\hline RE & $\begin{array}{l}\text { Сердечно-легочная недостаточность; } \\
\text { Фибрилляция предсердий }\end{array}$ & $\begin{array}{l}-0,875 \\
-0,576\end{array}$ & $\begin{array}{l}0,0004 \\
0,051\end{array}$ \\
\hline $\mathrm{MH}$ & $\begin{array}{l}\text { Условия глубокой гипотермии и краниоцеребральной гипотермии на фоне системной ОК; } \\
\text { Неврологические осложнения; } \\
\text { Время окклюзии аорты }\end{array}$ & $\begin{array}{l}-0,542 \\
-0,523 \\
-0,623\end{array}$ & $\begin{array}{l}0,032 \\
0,012 \\
0,061\end{array}$ \\
\hline PCS & $\begin{array}{l}\text { Сердечно-легочная недостаточность; } \\
\text { Фибрилляция предсердий }\end{array}$ & $\begin{array}{l}-0,721 \\
-0,494\end{array}$ & $\begin{array}{l}0,008 \\
0,036\end{array}$ \\
\hline MCS & $\begin{array}{l}\text { Сердечно-легочная недостаточность; } \\
\text { Фибрилляция предсердий }\end{array}$ & $\begin{array}{l}-0,628 \\
-0,494\end{array}$ & $\begin{array}{l}0,005 \\
0,008\end{array}$ \\
\hline
\end{tabular}

Сокращения: КЖ - качество жизни, динамика $(\Delta)$ показателей КЖ: ((параметр КЖ в отдаленные сроки после операции-исходный параметр КЖ)/исходный параметр КЖ) $100 \%$, PF - показатели шкалы физического функционирования, RF - показатели шкалы ролевого функционирования, ВР - показатели шкалы интенсивности боли, GH - показатели шкалы общего состояния здоровья, VT - показатели шкалы жизненной активности, SF - показатели шкалы социального функционирования, RE - показатели шкалы ролевого эмоционального функционирования, MH - показатели шкалы психического здоровья, РCS - показатели шкалы физического благополучия, MCS - показатели шкалы душевного благополучия, ОК - остановка кровообращения. 
общего состояния здоровья (GH) и показателя психического здоровья (МН).

Следует также подчеркнуть, что в отдаленном периоде после протезирования аорты наиболее низкие показатели КЖ (от 50 баллов и ниже) оставались по общему состоянию здоровья $(\mathrm{GH})$, жизненной активности (VT), а также по уровню физического и душевного благополучия (PCS, MCS).

С помощью многофакторного линейного регрессионного анализа выявлены клинические и интраоперационные факторы, влияющие на динамику КЖ в отдаленном периоде после протезирования аорты (табл. 3).

Как показано в таблице 3, на динамику параметров КЖ в отдаленном периоде после протезирования восходящего отдела и дуги аорты влияют как интраоперационные условия (метод церебральной защиты), так и течение раннего послеоперационного периода (развитие неврологических осложнений, сердечно-легочной недостаточности, фибрилляции предсердий). Такие показатели, как пол, возраст, масса тела, сопутствующая патология, вид протезирования восходящего отдела аорты (с сохранением аортального клапана либо с применением клапансодержащего кондуита), время нахождения в отделении интенсивной терапии, время искусственной вентиляции легких, по данным регрессионного анализа не оказывают значимого влияния на КЖ в отдаленный период после операции. Ограниченное количество факторов, влияющих на КЖ, возможно, связано c небольшой продолжительностью наблюдения в данном исследовании.

\section{Обсуждение}

Более полная оценка эффективности хирургического лечения с целью выбора оптимальной тактики реабилитации может быть произведена исследованием КЖ, поскольку это понятие является многофакторным, включая в себя экономические, психосоциальные, технологические и медицинские аспекты [8].

По данным нашего исследования, исходно тяжелая категория пациентов, обусловленная обширной площадью расслоения аорты, сопровождающегося выраженным болевым синдромом, способствует значительному снижению многих показателей КЖ. Через год после протезирования восходящего отдела и дуги аорты у пациентов отмечена положительная динамика по шкале болевого синдрома, что сопровождалось улучшением физического состояния, социальной адаптации и ролевого эмоционального функционирования. Тем не менее, оценка больными своего состояния здоровья (GH), энергичности (VT) оставалась на низком уровне (от 50 баллов и ниже). Несмотря на то, что показатели физического и психологического компонентов здоровья (PCS; MCS) имели положительную динамику, данные параметры в отда- ленные сроки после операции также не превышали 50 баллов.

При выполнении протезирования восходящего отдела и дуги аорты возникают не только технические сложности (длительность оперативного вмешательства, ИК), но и необходимость защиты центральной нервной системы во время ОК, так как все перечисленные факторы могут повлиять на КЖ данных пациентов [8]. Несмотря на удобство для хирурга выполнения системной гипотермической ОK (“сухое” операционное поле, хороший доступ), данный метод имеет свои недостатки. Это неадекватная доставка компонентов метаболизма к головному мозгу и невозможность в достаточной мере обеспечить церебральную защиту, а также увеличение времени операции за счет длительного охлаждения/ согревания [9]. В нашем исследовании протезирование проксимального отдела аорты в условиях глубокой гипотермии и краниоцеребральной гипотермии на фоне системной ОK, а также развитие неврологических осложнений в госпитальный период, оказывали отрицательное влияние на динамику таких параметров КЖ, как общее состояние здоровья, психическое здоровье и душевное благополучие. На динамику многих параметров КЖ, включая суммарные шкалы физического и душевного благополучия, отрицательное влияние оказывали развитие в ранний послеоперационный период фибрилляции предсердий и сердечно-легочной недостаточности.

В наиболее ранних работах изучались показатели КЖ у пациентов после протезирования грудного отдела аорты в зависимости от интраоперационных условий, где с увеличением продолжительности ОК отмечалось ухудшение параметров физического и психического благополучия. В этой же работе было выявлено, что применение во время ОК АПГМ связано с улучшением показателей КЖ после операции [10], что согласуется с результатами нашего исследования. В более поздних исследованиях было отмечено, что наличие инфаркта миокарда в анамнезе и пожилой возраст способствовали снижению показателя физического благополучия после протезирования проксимального отдела аорты. При этом, проведя многофакторный регрессионный анализ, авторы не обнаружили влияния на КЖ продолжительности ОК или применения АПГМ [11]. В нашем исследовании пол, возраст, масса тела, сопутствующая патология, вид протезирования восходящего отдела аорты по данным регрессионного анализа не оказывают значимого влияния на параметры КЖ в отдаленный период после операции, что, возможно, связано с небольшой продолжительностью наблюдения.

\section{Заключение}

Таким образом, по данным нашего исследования, клиническими и интраоперационными факторами, 
неблагоприятно влияющими на динамику различных показателей КЖ через 12 мес. после протезирования восходящего отдела и дуги аорты явились церебральная защита в условиях глубокой гипотермии и краниоцеребральной гипотермии на фоне системной ОК; развитие неврологических осложнений, а также фибрилляция предсердий и сердечно-легочная недостаточность в ранний послеоперационный период.

Выявление факторов, влияющих на динамику КЖ в послеоперационный период у пациентов с расслое-

\section{Литература/References}

1. Erbel R, Aboyans V, Boileau C, et al. ESC Committee for Practice Guidelines. Guidelines on the diagnosis and treatment of aortic diseases: Document covering acute and chronic aortic diseases of the thoracic and abdominal aorta of the adult. The Task Force for the Diagnosis and Treatment of Aortic Diseases of the European Society of Cardiology (ESC). Eur Heart J. 2014;35:41:2873-926. doi:10.1093/eurheartj/ehu281.

2. Ljahova NL. Long-term results and the quality of life of patients after surgical treatment of dissecting aneurysms of the ascending aorta with the transition to the arc. Annaly hirurgii. 2009; 3: 9-15. (In Russ.) Ляхова Н. Л. Отдаленные результаты и качество жизни пациентов после хирургического лечения расслаивающих аневризм восходящей аорты с переходом на дугу. Анналы хирургии. 2009;3:9-15.

3. Kamenskaya OV, Cherniavsky AM, Klinkova AS, et al. Efficiency of various cerebral protection techniques used during the surgical treatment of chronic pulmonary thromboembolism. J Extra Corpor Technol. 2015;47:2:95-102.

4. Belov YuV, Charchyan ER, Akselrod BA, et al. Cerebral and visceral organ protection during aortic arch surgery. Intraoperative tactics and monitoring details. Patologiya krovoobrashcheniya i kardiokhirurgiya (Circulation Pathology and Cardiac Surgery). 2016;20,4:34-44. (In Russ.) Белов Ю.В., Чарчян Э. Р., Аксельрод Б.А. и др. Защита головного мозга и внутренних органов при реконструктивных вмешательствах на дуге аорты: особенности интраоперационной тактики и мониторинга. Патология кровообращения и кардиохирургия. 2016;20,4:34-44. doi:10.21688/1681-3472-20164-34-44.

5. Klinkova AS, Kamenskaya OV, Cherniavsky AM, et al. The risk of developing neurological complications in prosthetics of the ascending department and the aortic arch. Angiology нием восходящего отдела и дуги аорты, позволит улучшить эффективность хирургического лечения путем создания оптимальной программы реабилитации.

Финансирование. Работа выполнена при финансовой поддержке гранта РНФ № 17-75-30009.

Конфликт интересов: все авторы заявляют об отсутствии потенциального конфликта интересов, требующего раскрытия в данной статье.

and Vascular Surgery. 2017;23,1:124-9. (In Russ.) Клинкова А.С., Каменская О.В., Чернявский А.М. и др. Риск развития неврологических осложнений при протезировании восходящего отдела и дуги аорты. Ангиология и сосудистая хирургия. 2017;23,1:124-9.

6. Lohse F, Lang N, Schiller W, et al. Quality of Life after Replacement of the Ascending Aorta in Patients with True Aneurysms. Tex Heart Inst J. 2009;36,2:104-10.

7. Data processing instructions obtained by the SF- 36 questionnaire. Electronic resource. Company Evidence - URL://http://therapy.irkutsk.ru/doc/sf36a.pdf (date of the application: 19.08.2014.

8. Hamiko M, Gestrich C, Probst C, et al. Long-term outcome and quality of life in aortic type A dissection survivors. Thorac and Cardiovasc Surg. 2015;64,2:91-9. doi:10.1055/s-0035-1548734.

9. Sahu B, Chauhan S, Kiran U, et al. Neurocognitive function in patients undergoing coronary artery bypass surgery with cardiopulmonary bypass: the effect of two different rewarming strategies. J Cardiothor Vasc Anaesth. 2009;23,1:14-21. doi:10.1053/j.jvca.2008.07.010.

10. Immer FF, Lippeck $\mathrm{C}$, Barmettler $\mathrm{H}$, et al. Improvement of quality of life after surgery on the thoracic aorta. Effect of antegrade cerebral perfusion and short duration of deep hypothermic circulatory arrest. Circulation. 2004;110,11:11250-55. doi:10.1161/01. cir.0000138387.61103.a0.

11. Olsson C, Franco-Cereceda A. Health-Related Quality of Life in Thoracic Aortic Disease Part II. After Surgery on the Proximal (Root, Ascending, Arch) Aorta. Aorta. 2013;1,3:16270. doi:10.12945/j.aorta.2013.13-029. 